\title{
MHD Heat and Mass Transfer Forced Convection Flow along a Vertical Stretching Sheet with Heat Generation and Radiation
}

\author{
M. A. Samad and S. Ahmed \\ Department of Mathematics, University of Dhaka, Dhaka-1000, Bangladesh.
}

\begin{abstract}
The present study comprises of steady two dimensional magnetohydrodynamic heat and mass transfer forced convection flow along a vertical stretching sheet in the presence of magnetic field with radiation. The nonlinear partial differential equations governing the flow field occurring in the problem have been transformed to dimensionless nonlinear ordinary differential equations by introducing suitably selected similarity variables. The ensuing equations are simultaneously solved by applying Nachtsheim-Swigert shooting iteration technique with sixth order Runge-Kutta integration scheme. The results in the form of velocity, temperature and concentration profiles are then displayed graphically. The corresponding skin-friction coefficient, Nusselt number and Sherwood number are displayed graphically and also in tabular form as well. Several important parameters such as the prandtl number $(\operatorname{Pr})$, radiation parameter $(N)$, magnetic field parameter $(M)$, heat source parameter $(Q)$, schmidt number $(S c)$, suction parameter $\left(f_{w}\right)$ and eckert number $(E c)$ are confronted. The effects of these parameters on the velocity, temperature and concentration profiles are investigated.
\end{abstract}

Key Words: MHD, Forced convection, Stretching sheet, Radiation, Heat generation.

\section{Introduction}

Out of two types of convective heat and mass transfer, forced convection is the one where the velocity of the flow dominates over the other parameters. Some kind of external forces are employed here. The cooling system in a car engine is an example of forced convection. Thermal radiation effects may play an important role in controlling heat transfer in industry where the quality of the final product depends on the heat controlling factors to some extent. High temperature plasmas, cooling of nuclear reactors, liquid metal fluids, and power generation systems are some important applications of radiative heat transfer from a vertical wall to conductive gray fluids. Thermal radiation effect on forced and free convection have been studied in recent years extensively, as the magnetohydrodynamic [MHD] flow and heat transfer problems have become more important in many engineering and industrial applications. Hossain and Takhar (1996) studied the radiation effects using the Rosseland diffusion approximation (Seigel and Howell, 1972) that leads to non similar boundary layer equation governing the mixed convection flow of an optically dense viscous incompressible fluid past heated vertical plate with a free uniform velocity and surface temperature. Shateyi et al. (2008) studied the thermal radia-

\footnotetext{
* Corresponding author: E-mail: mdabduss@yahoo.com
}

tion and buoyancy effects on heat and mass transfer over a semi-infinite stretching surface with suction and blowing. Ali et al. (1984) studied the radiation effect on natural convection flow over a vertical surface in a gray gas. Following Ali et al. Mansour (1990) studied the interaction of mixed convection with thermal radiation in laminar boundary layer flow over a horizontal, continuous moving sheet with suction / injection. Elbashbeshy et al. (2000) studied the determination of the effect of radiation on forced convection flow of a micropolar fluid over a horizontal plate. Aydin et al. (2008) investigated the mixed convection heat transfer about a permeable vertical plate in the presence of magneto and thermal radiation effects. Cess (1966) studied to determine the influence of radiation heat transfer upon the forced convection Nusselt number.

Samad and Rahman (2006) investigated thermal radiation interaction with unsteady MHD flow past a vertical porous plate immersed in a porous medium. Samad and Karim (2009) studied thermal radiation interaction with unsteady MHD free convection flow through a vertical flat plate with time dependent suction in the presence of magnetic field. As 
in the case of stretching sheets, Chen (1998) studied laminar mixed convection adjacent to vertical continuously stretching sheet. Chiam (1997) investigated magnetohydrodynamic heat transfer over non-isothermal stretching sheet. Pop et al. (2004) studied radiation effect on the flow near the stagnation point of a stretching sheet. Abo-eldahab (2005) studied flow and heat transfer in a micropolar fluid past a stretching surface embedded in a non-Darcian porous medium with uniform free stream. In the present study thermal radiation interaction on MHD forced convection flow in the presence of a uniform magnetic field over a stretching sheet has been investigated.

\section{Mathematical Formulation}

We consider a steady two-dimensional magnetohydrodynamic heat and mass transfer flow of a viscous incompressible fluid along a vertical stretching sheet with constant heat generation/absorption with radiation.

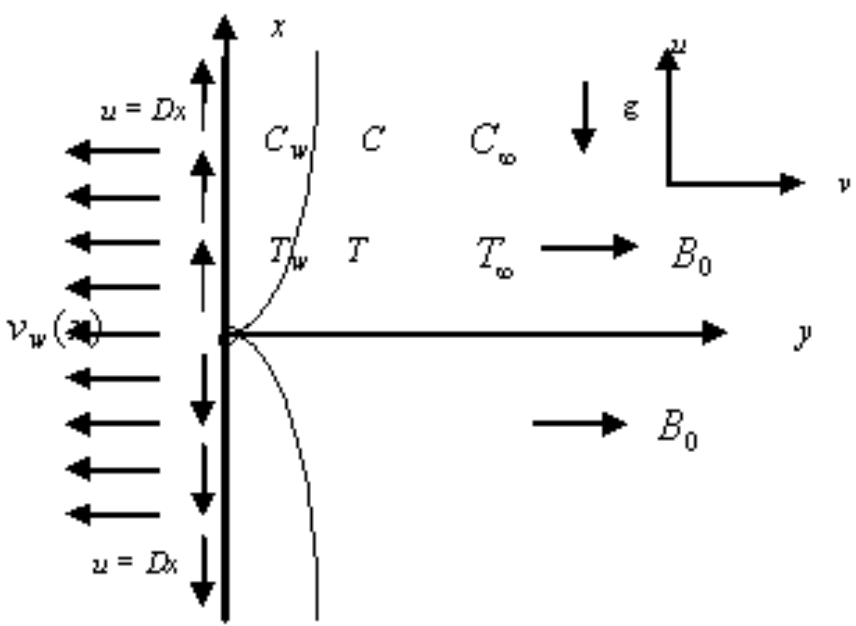

Fig. 1: Flow configuration and coordinate system.

We take the $\mathrm{x}$-axis along the sheet and $\mathrm{y}$-axis perpendicular to it. Two equal and opposite forces are introduced along the $\mathrm{x}$-axis so that the sheet is stretched keeping the origin fixed. A uniform magnetic field of strength $B_{0}$ is imposed along the y-axis. A radiation depending on temperature is applied on the stretching sheet.

The governing equations representing the proposed flow field are:

Continuity Equation:

$$
\frac{\partial u}{\partial x}+\frac{\partial v}{\partial y}=0
$$

Momentum Equation:

$u \frac{\partial u}{\partial x}+v \frac{\partial u}{\partial y}=v \frac{\partial^{2} u}{\partial y^{2}}+\operatorname{sg} \beta\left(T-T_{\infty}\right)-\frac{\sigma B_{0}^{2}}{\rho} u$

Energy Equation:

$$
\begin{aligned}
& u \frac{\partial T}{\partial x}+v \frac{\partial T}{\partial y}=\frac{\kappa}{\rho C_{p}} \frac{\partial^{2} T}{\partial y^{2}} \\
& +\frac{Q_{0}}{\rho C_{p}}\left(T-T_{\infty}\right)-\frac{1}{\rho C_{p}} \frac{\partial q_{r}}{\partial y}
\end{aligned}
$$

Concentration Equation:

$u \frac{\partial C}{\partial x}+v \frac{\partial C}{\partial y}=D_{m} \frac{\partial^{2} C}{\partial y^{2}}$

Here $\mathrm{u}$ and $\mathrm{v}$ are the velocity components along the $\mathrm{x}$ and $\mathrm{y}$ directions respectively, $v$ is the kinematic viscosity, $\mathrm{g}$ is the acceleration due to gravity, $\beta$ is the volumetric coefficient of thermal expansion, $B_{0}$ is the uniform magnetic field strength (magnetic induction), $T$ and $T_{\mathrm{oo}}$ are the fluid temperature within the boundary layer and in the free stream respectively, $\mathrm{C}$ is the concentration of the fluid within the boundary layer, $\sigma$ is the electric conductivity, $\rho$ is the density of the fluid, $k$ is the thermal conductivity of the fluid, $C_{p}$ is the specific heat at constant pressure, $Q_{0}$ is the volumetric rate of heat generation/ absorption and $D_{m}$ is the chemical molecular diffusivity, $s$ is a dummy parameter stands for 0 (forced convection), +1 (heating problem) and -1 (cooling problem).

\section{Boundary conditions}

The boundary conditions can be split into two parts. The first is the wall boundary conditions and the second is the free stream conditions.

\section{Wall boundary conditions}

$u=D x, v=v_{w}, T=T_{w}, C=C_{w} \quad$ at $\quad y=0$

Free stream conditions

$u=0, T=T_{\infty}, C=C_{\infty} \quad$ as $\quad y \rightarrow \infty$

Here, $\mathrm{D}(>0)$ is a constant, $v_{w}$ is a velocity component at the wall having positive value to indicate suction. $T_{w}$ is the uni- 
form wall temperature and $C_{w}, C_{\mathrm{oo}}$ are the concentration of the fluid at the sheet and far from the sheet respectively.

The effect of the second term on the right hand side of the equation (2) is due to buoyancy effect on the flow field and the governing equations (1)-(4) represent forced convection flow when we take $s=0$.

We use Rosseland approximation to write $q_{r}$ in the following form

$q_{r}=-\frac{4 \sigma_{1}}{3 k_{1}} \frac{\partial T^{4}}{\partial y}$

where, $\sigma_{1}$ is the Stefan-Boltzmann constant and $k_{1}$ is the mean absorption coefficient.

It is assumed that the temperature differences within the flow are sufficiently small such that $T^{4}$ can be expanded in a Taylor series about $T_{\mathrm{oo}}$ and neglecting higher-order terms, thus

$T^{4} \approx 4 T_{\infty}^{3} T-3 T_{\infty}^{4}$

Using (5) and (6) in equation (3) we have

$u \frac{\partial T}{\partial x}+v \frac{\partial T}{\partial y}=\frac{\kappa}{\rho C_{p}} \frac{\partial^{2} T}{\partial y^{2}}$

$+\frac{Q_{0}}{\rho C_{p}}\left(T-T_{\infty}\right)+\frac{16}{3} \frac{\sigma_{1} T_{\infty}^{3}}{\rho C_{p} k_{1}} \frac{\partial^{2} T}{\partial y^{2}}$

Summarizing the problem we obtain the following system:

$$
\begin{aligned}
& \frac{\partial u}{\partial x}+\frac{\partial v}{\partial y}=0 \\
& u \frac{\partial u}{\partial x}+v \frac{\partial u}{\partial y}=v \frac{\partial^{2} u}{\partial y^{2}}-\frac{\sigma B_{0}^{2}}{\rho} u
\end{aligned}
$$

$u \frac{\partial T}{\partial x}+v \frac{\partial T}{\partial y}=\frac{\kappa}{\rho C_{p}} \frac{\partial^{2} T}{\partial y^{2}}$

$+\frac{Q_{0}}{\rho C_{p}}\left(T-T_{\infty}\right)+\frac{16}{3} \frac{\sigma_{1} T_{\infty}^{3}}{\rho C_{p} k_{1}} \frac{\partial^{2} T}{\partial y^{2}}$

$u \frac{\partial C}{\partial x}+v \frac{\partial C}{\partial y}=D_{m} \frac{\partial^{2} C}{\partial y^{2}}$
The boundary conditions as a whole are

$$
\left.\begin{array}{ll}
u=D x, \quad v=v_{w}, \quad T=T_{w}, \quad C=C_{w} & \text { at } y=0, \\
u=0, \quad T=T_{\infty}, \quad C=C_{\infty} & \text { as } y \rightarrow \infty
\end{array}\right\}
$$

The following similarity variables are introduced to obtain a similarity solution corresponding to the above problem.

$$
\begin{aligned}
& M=\frac{\sigma B_{0}^{2}}{\rho D} \\
& \psi=\sqrt{D v} x f(\eta) \\
& \theta(\eta)=\frac{T-T_{\infty}}{T_{w}-T_{\infty}} \\
& \phi(\eta)=\frac{C-C_{\infty}}{C_{w}-C_{\infty}}
\end{aligned}
$$

Here $\Psi$ is the stream function, $\mathrm{h}$ is the dimensionless distance normal to the sheet, $f$ is the dimensionless stream function, $q$ is the dimensionless fluid temperature and $f$ is the dimensionless concentration.

Since $u=\frac{\partial \psi}{\partial y}$ and $v=-\frac{\partial \psi}{\partial x}$ we have the velocity components from equation (14) given by

$$
\left.\begin{array}{l}
u=D x f^{\prime}(\eta) \\
v=-\sqrt{D v} f(\eta)
\end{array}\right\}
$$

Here prime denotes the derivatives with respect to $\eta$.

Now substituting the similarity variables from equations (12) to (17) into the momentum equation (9), (10) and (11) we have the system as,

$$
\begin{aligned}
& f^{\prime \prime \prime}+f f^{\prime \prime}-\left(f^{\prime}\right)^{2}-M f^{\prime}=0 \\
& \theta^{\prime \prime}+\frac{3 N \operatorname{Pr}}{3 N+4} f \theta^{\prime}+\frac{3 N \operatorname{Pr} Q}{3 N+4} \theta=0 \\
& \phi^{\prime \prime}+S c f \phi^{\prime}=0
\end{aligned}
$$


The transformed boundary conditions are:

$$
\left.\begin{array}{l}
f^{\prime}=1, \quad f=f_{w}, \quad \theta=1, \quad \phi=1 \quad \text { at } \eta=0 \\
f^{\prime}=0, \quad \theta=0, \quad \phi=0 \quad \text { as } \eta \rightarrow \infty
\end{array}\right\}
$$

Here, $\quad M=\frac{\sigma B_{0}^{2}}{\rho D} \quad$ is the magnetic field parameter, $\operatorname{Pr}=\frac{\mu C_{p}}{\kappa}$ is the Prandtl number, $Q=\frac{Q_{0}}{\rho C_{p} D}$ is the $\kappa$
heat source/ sink parameter, $N=\frac{\kappa k_{1}}{4 \sigma_{1} T_{\infty}^{3}}$ is the radiation parameter and, $f_{w}=-\frac{v_{w}}{\sqrt{D v}}$ is the suction parameter.

\section{Numerical computations}

The nonlinear ordinary differential equations (18), (19) and (20) under the boundary conditions (21) are solved numerically for various values of the parameters occurring in the problem. Here we use the standard initial-value solver shooting method namely Nachtsheim-Swigert (1965) shooting iteration technique (guessing the missing value) together with the sixth order Runge-Kutta-Butcher initial value solver. A step size of $\mathrm{Dh}=0.001$ has been used together with accuracy $10^{-6}$ of in all the cases. The value of $h_{\max }$ was selected in accordance with the values of each group of parameters $\operatorname{Pr}, f_{w}, M, Q$ and $N$ to satisfy the accuracy requirement. The code verifying graphs for different step sizes are shown below.

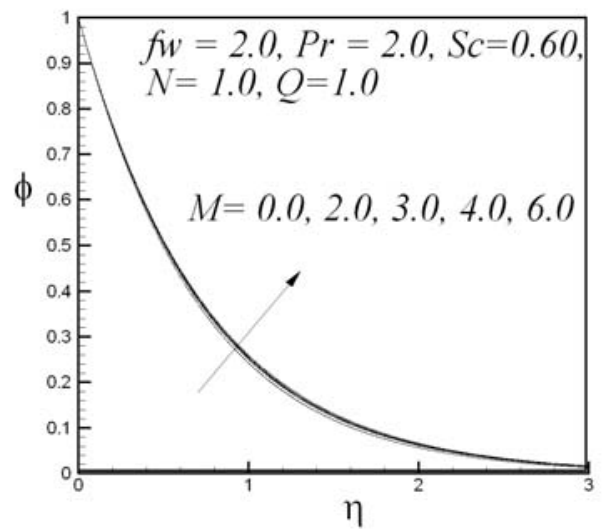

Fig. 2(a): Velocity profiles for various step sizes

Here, we see that for step sizes, $\mathrm{Dh}=0.01,0.005,0.001$ the velocity and the temperature profiles are in good agreement among them.

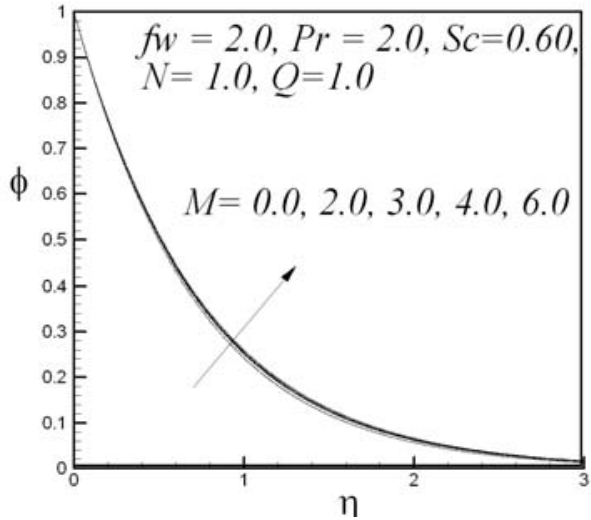

Fig. 2(b): Temperature profiles for various step sizes

\section{Results and Discussion}

We use the dimensionless velocity, temperature, and concentration profiles to present the results obtained in the numerical computations. Numerical computations have been carried out for various values of parameters entering into the problem in compliance with the different physical conditions. These parameters are, the Prandtl number $(\mathrm{Pr})$, suction parameter $\left(f_{w}\right)$, magnetic field parameter $(M)$, radiation parameter $(N)$, Schmidt number $\left(S_{c}\right)$ and heat source parameter $(Q)$.

The temperature profiles show a sharp variation in the Fig. 3. With the increase of Prandtl number the decreasing rate of the temperature increases. That is, the heat transfer rate in the flow field increases with the increase of the Prandtl number. This is because, for lower Prandtl number, for example $\operatorname{Pr}=0.71$, which represents air, the heat is transferred at much slower rate. On the other hand for $\mathrm{Pr}=7.0$, representing water at $20^{\circ} \mathrm{C}$, the heat is transformed at much higher rate. The thermal boundary layer thickness decreases to a large extent with the increase of the Prandtl number.

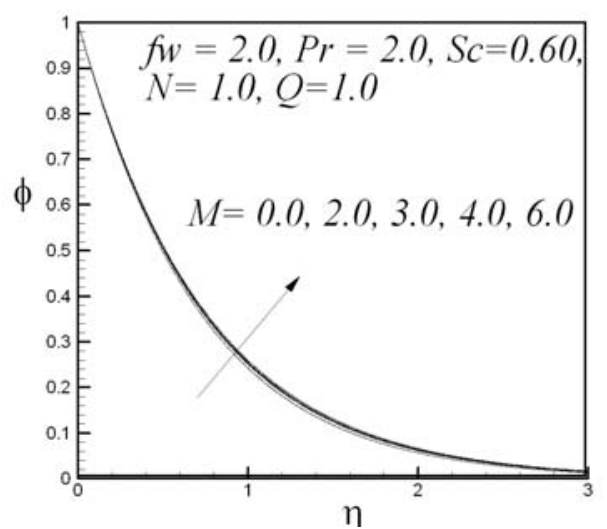

Fig. 3:Temperature profiles for various values of prandtl number, Pr. 
Fig. 4 shows the effect of radiation on the velocity, temperature, and concentration profiles. Since radiation is in the form of electromagnetic waves, no effects are seen on the velocity profiles (Fig. 4(a)). It is because of the higher velocity in the forced convection. The momentum boundary layer thickness remains fixed with the increase of the radiation parameter. The temperature profiles show that, with the increase of radiation flux, the temperature in the vicinity of the boundary layer decreases more rapidly (Fig. 4(b)). This can be explained easily by the fact that, with the increase of the radiation the heat transfer rate must increase. There is a large difference in the temperature profiles between the values 0.10 and 0.50 for $N$. This is because, at the value of 0.10 the radiation is very negligible and upon reaching the value of 0.50 , it becomes significant in the temperature field in the thermal boundary layer. With the increase of the radiation parameter the thermal boundary layer thickness reduces very rapidly. In the Fig. 5 the effect of magnetic field parameter, $M$ is demonstrated. The Fig. 5(a) shows that a variation occurs in the velocity profiles, that is, the rate of decrease, increases with the increase of the magnetic field parameter; $M$.

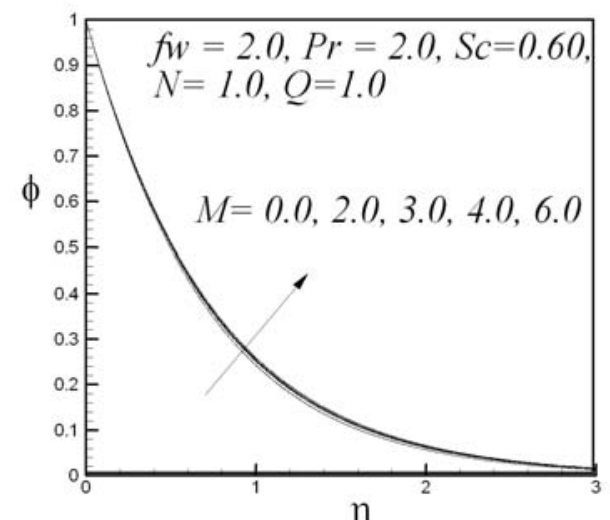

Fig. 4(a): Velocity profiles for various values of radiation parameter, $N$

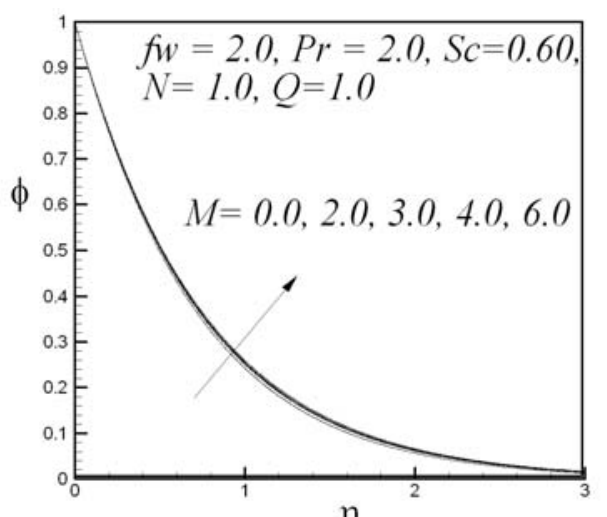

Fig. 4(b): Temperature profiles for various values of radiation parameter, $N$
It can be explained by the fact that the magnetic field produces a retarded action on the velocity field, thus decreasing the velocity at a higher rate. The momentum boundary layer thickness decreases slightly with the increase of the magnetic parameter. Fig. 5(b) shows a slight decrease in the

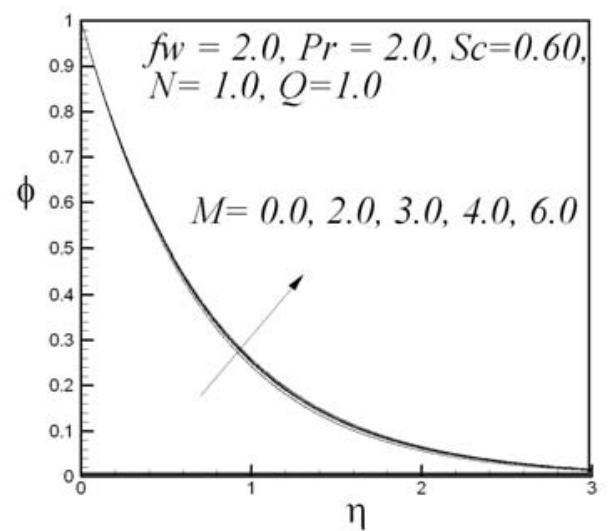

Fig. 5(a): Velocity profiles for various values of magnetic parameter, $M$

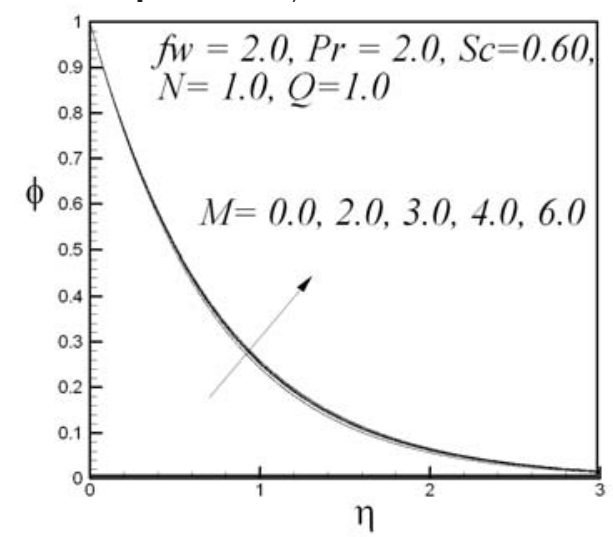

Fig. 5(b): Temperature profiles for various values of magnetic parameter, $M$

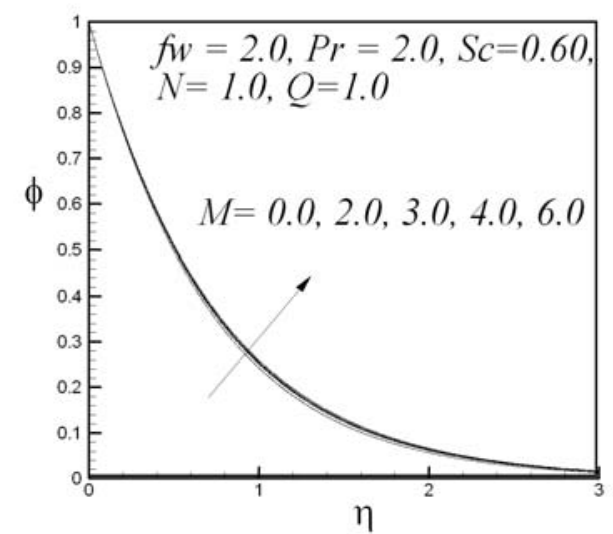

Fig. 5(c): Concentration profiles for various values of magnetic parameter, $M$ 
decreasing rate of the temperature profiles. That is, the heat transfer rate decreases with the increase in the magnetic field strength. This is explained due to the retarding action of the magnetic field on the boundary layer causing the heat transfer rate getting slower and slower. This implies that the thermal boundary layer thickness increases a small amount with the increase of the magnetic parameter. The concentration profiles in the Fig. 5(c) also show a similar pattern of decreasing transfer rate due to the magnetic field effect.

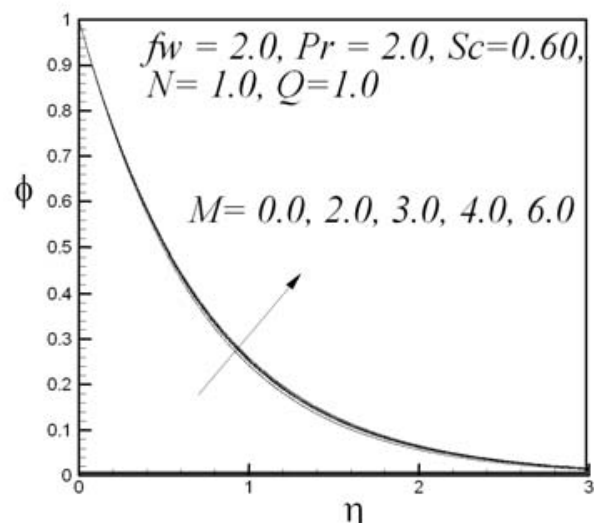

Fig. 6: Concentration profiles for various values of Schmidt number, $S c$

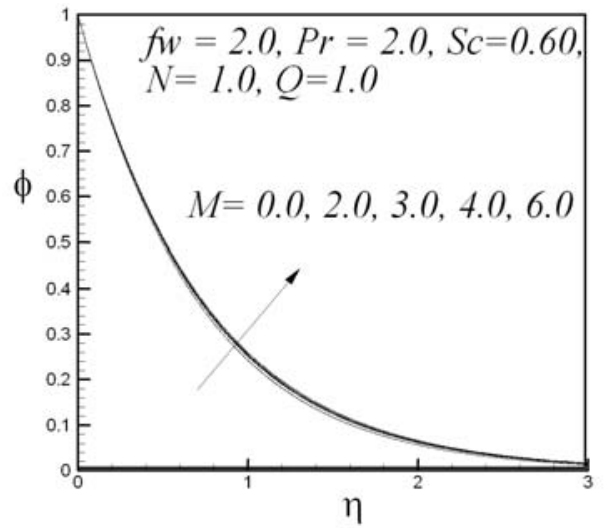

Fig. 7: Temperature profiles for various values of heat source parameter, $Q$

The concentration profiles show a strong variation with the increase of Schmidt number (Fig. 6). The decreasing rate of the concentration profiles increase to a large extent due to the increase in the Schmidt number. This is because with the increase of the Schmidt number the kinematic viscosity increases and the mass diffusion coefficient decreases causing the concentration decrease rapidly. The temperature profiles show a decrease in the decreasing rate of heat source
(Fig. 7) in accordance with the physical requirements. The increase in the heat source parameter causes the temperature to decrease at a slower rate. This can be explained due to the fact that the heat source resists the temperature to decrease rapidly. In the case of a high heat source, for example, $Q=$ 7.0, the temperature rises at first and then decreases to the free stream temperature. The thermal boundary layer increases a great amount with the increase of the heat source parameter.

In the Fig. 8 the effect of suction is represented. We see that in all the three cases of velocity, temperature and concentration profiles there is sharp variation in the curves. The velocity profiles show an increase in their decreasing rate with the increase of the suction in the Fig. 8(a). This can be explained by the fact that when the suction parameter increases, some of matter is removed from the system and the velocity gets retarded more rapidly. Also the momentum boundary layer

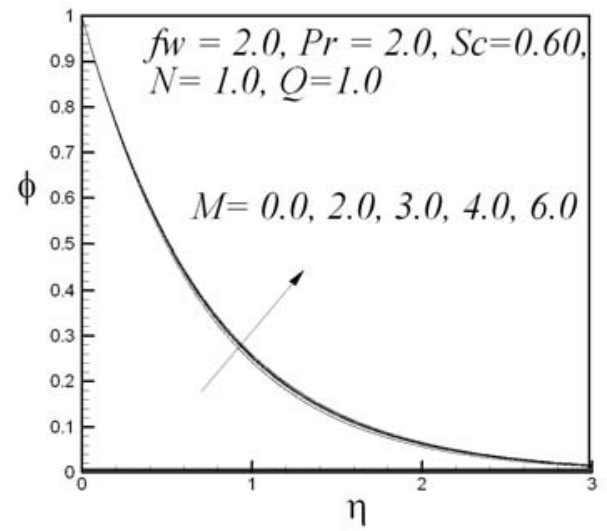

Fig. 8(a): Velocity profiles for various values of suction parameter, $f_{w}$

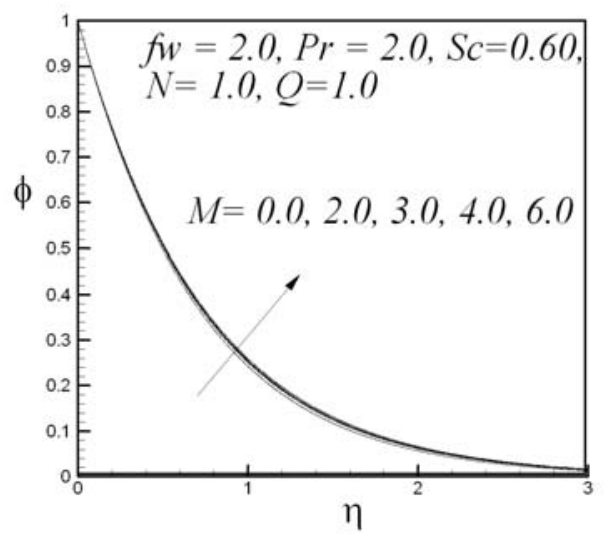

Fig. 8(b): Temperature profiles for various values of suction parameter, $f_{w}$ 
thickness decreases with the increase of the suction parameter and thus reduces the chance of the boundary layer to the transition to turbulence. The temperature profiles in the Fig. 8(b) shows also a decreasing trend with the increase of the suction parameter. Thus it reduces the thermal boundary layer thickness with the increase of the suction parameter to a significant amount.

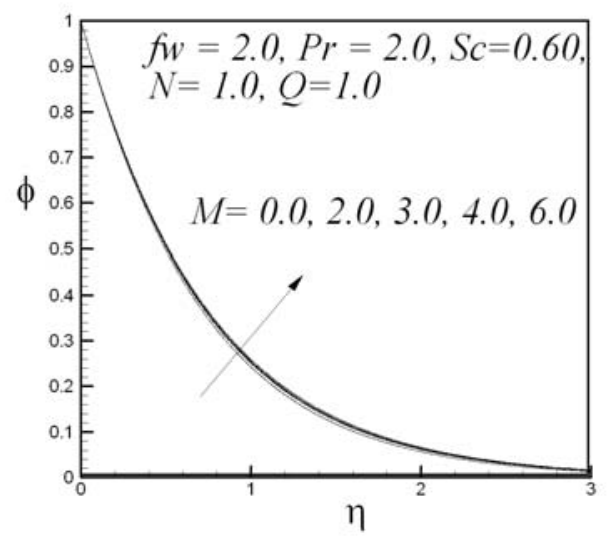

Fig. 8(c): Concentration profiles for various values of suction parameter, $f_{w}$

The concentration profiles in the Fig. 8(c) shows a large variation on the effect of suction parameter. As the two other cases the concentration profiles also show a large amount of decreasing trend with the increase of the suction parameter.

\section{Skin-Friction Coefficient, Nusselt Number, and} Sherwood Number

The skin friction coefficient $\left(C_{f}\right)$, local Nusselt number $\left(N u_{x}\right)$ and the local Sherwood numbers $(S h)$ are significant in the engineering field. These parameters refer to the wall shear stress, local wall heat transfer rate and wall mass transfer rate respectively. It is observed that the skin-friction coefficient, Nusselt number, and Sherwood number are proportional to $f^{\prime \prime}(0),-\theta^{\prime}(0)$, and $-\phi^{\prime}(0)$ respectively. Tables comprising of the proportional values of $C_{f}, N u_{x}$, and $S h$ corresponding to the previous graphs are shown below.

Table I: $C_{f}, N u_{x}$, and $S h$ for different values of $\mathrm{Pr}$

\begin{tabular}{l|c|c|c}
\hline $\operatorname{Pr}$ & $C_{f}$ & $N u_{x}$, & $S h$ \\
\hline 0.71 & 5.28387356 & 1.31886196 & 1.1352173 \\
1.0 & 5.28387403 & 2.20613384 & 1.1352621 \\
2.0 & 5.28387403 & 4.38756895 & 1.1352621 \\
5.0 & 5.28387403 & 10.8617086 & 1.1352621 \\
7.0 & 5.28387403 & 14.9462938 & 1.1352621 \\
\hline
\end{tabular}

Table II: $C_{f}, N u_{x}$, and $S h$ for different values of $N$

\begin{tabular}{l|c|c|c}
\hline $\mathrm{N}$ & $C_{f}$ & $N u_{x}$, & $S h$ \\
\hline 0.10 & 5.28386784 & 0.40335795 & 1.1342582 \\
0.50 & 5.28387403 & 2.58753276 & 1.1352621 \\
1.0 & 5.28387403 & 4.17267227 & 1.1352621 \\
2.0 & 5.28387403 & 5.90563250 & 1.1352621 \\
5.0 & 5.28387403 & 7.81462622 & 1.1352621 \\
\hline
\end{tabular}

Table III: $C_{f}, N u_{x}$, and $S h$ for different values of $M$

\begin{tabular}{l|c|c|c}
\hline $\mathrm{M}$ & $C_{f}$ & $N u_{x}$, & Sh \\
\hline 0.00 & 2.41421103 & 1.35434055 & 1.3584946 \\
2.00 & 3.00000000 & 1.29453099 & 1.3383700 \\
3.00 & 3.23606586 & 1.27346361 & 1.3315948 \\
4.00 & 3.44948983 & 1.25515652 & 1.3259624 \\
6.00 & 3.82842660 & 1.22583878 & 1.3170682 \\
\hline
\end{tabular}

Table IV: $C_{f}, N u_{x}$, and $S h$ for different values of $Q$

\begin{tabular}{l|c|c|c}
\hline $\mathrm{Q}$ & $C_{f}$ & $N u_{x}$, & $S h$ \\
\hline 0.00 & 5.37227821 & 4.37344217 & 1.1348044 \\
2.00 & 5.37227821 & 3.94760203 & 1.1348044 \\
4.00 & 5.37227821 & 3.38222218 & 1.1348044 \\
6.00 & 5.37227821 & 1.41797698 & 1.1348044 \\
7.00 & 5.37227821 & -0.3556116 & 1.1348044 \\
\hline
\end{tabular}

TableV: $C_{f}, N u_{x}$, and $S h$ for different values of $f_{w}$

\begin{tabular}{l|c|c|c}
\hline$f w$ & $C_{f}$ & $N u_{x}$, & $S h$ \\
\hline 1.0 & 1.82287526 & 2.65054321 & 0.3231116 \\
2.0 & 2.58113670 & 5.85618830 & 0.5119724 \\
3.0 & 3.43648672 & 8.91013527 & 0.7139405 \\
4.0 & 4.34520149 & 11.93455510 & 0.9227340 \\
5.0 & 5.28387403 & 14.94845390 & 1.1352621 \\
\hline
\end{tabular}

\section{Conclusion}

The problem has dealt with the two dimensional heat and mass transfer forced convection flow of an MHD fluid along a stretching sheet in the presence of magnetic field with heat generation and radiation.

We can make the following conclusions:

1. In the forced convection the velocity being large, the Prandtl number has no effective dominance over velocity and concentration. Nevertheless the temperature it has a significant effect. With the increase of Prandtl number the local heat transfer rate increases. 
2. The radiation parameter has a large effect on the temperature profiles, and hence can be used effectively to control the temperature of the flow field.

3. The magnetic field parameter has significant effect on the velocity, temperature and concentration profiles. The local skin-friction coefficient, heat transfer rate and mass transfer rate decrease with the increase of the magnetic parameter. So, magnetic field can effectively be used to control the flow field.

4. As apparent the temperature increases within the boundary layer with the increase of the heat source parameter.

5. For the increase of the suction parameter the velocity, temperature and concentration significantly decrease and as a consequence making it one of the most essential parameter in the boundary layer control theory.

\section{References}

Abo-Eldahab, E. M. (2005.). Flow And Heat Transfer in a Micropolar Fluid past a Stretching Surface Embedded in a Non-Darcian Porous Medium with Uniform Free Stream. Appl. Math. Comp. 162: 881-889.

Ali M. M., Chen, T. S. and Armaly B. F. (1984). Natural Convection Radiation Interaction In Boundary Layer Flow over Horizontal Surfaces. AIAA Journal, 22(12): 797-803.

Aydin O. and Kaya A. (2008). Radiation Effect On MHD Mixed Convection Flow About A Permeable Vertical Plate. Heat Mass Trans. 45: 239-246.

Cess R. D. (1966). The Effect of Radiation upon Forced Convection Heat Transfer, Appl. Sci. Res. Sec A. 10: 1269-1277.

Chen C. H. (1998). Laminar Mixed Convection Adjacent to Vertical, Continuously Stretching Sheet. Heat and Mass Tran. 33:471-476.
Chiam T. C. (1997). Magnetohydrodynamic Heat Transfer over a Non-Isothermal Stretching Sheet. Acta Mechanica, 122:169-179.

Elbashbeshy E. M. A. and Bazid M. A. A. (2000.). Effect of Radiation on Forced Convection Flow of a Micropolar Fluid over a Horizontal Plate. Can. J. Phys. 78: 907913.

Hossain, M. A. and Takhar H. S. (1996.). Radiation Effect on Mixed Convection along a Vertical Plate with Uniform Surface Temperature. Heat and Mass Trans.31(4): 243-248.

Mansour M. A. (1990.). Radiative and Free Convection Effects on the Oscillatory Flow past a Vertical Plate. Astrophysics Space Sci. 166: 269-275.

Nachtsheim P. R. and Swigert P. (1965). Satisfaction of the Asymptotic Boundary Conditions in Numerical Solution of the System of Non-Linear Equations of Boundary Layer Type. NASA TND-3004.

Pop. S.R., Grosan T. and Pop, I. (2004). Radiation Effect on the Flow near the Stagnation Point of a Stretching Sheet. The Chnische Mechanik, 25:100-106, 2004.

Samad, M. A. and Karim, M. E. (2009). Thermal Radiation Interaction with Unsteady MHD Flow past a Vertical Flat Plate with Time Dependent Suction. Dhaka Univ. J. Sci. 57(1): 113-118.

Samad M. A. and Rahman M. M. (2006). Thermal Radiation Interaction with Unsteady MHD Flow past a Vertical Porous Medium. J. Nav. Arch. Mar. Eng. 3:7-14.

Shateyi S. and Petersen M. (2008). Thermal Radiation and Buoyancy Effects on Heat and Mass Transfer over a Semi-Infinite Stretching Surface with Suction and Blowing. J. Appl. Math. 2008:12

Seigel R. and Howell J. R.(1972). Thermal Radiation Heat Transfer. McGraw-Hill New York.

Received: January, 10, 2010;

Accepted : November 25, 2010 